\title{
Parameterization of Quasigeostrophic Eddies in Primitive Equation Ocean Models
}

\author{
A. M. TReguier \\ LPO, IFREMER, Brest, France
}

I. M. HELD

GFDL/NOAA, Princeton, New Jersey

V. D. LARICHEV

AOS Program, Princeton University, Princeton, New Jersey

(Manuscript received 26 January 1996, in final form 26 September 1996)

\section{ABSTRACT}

\begin{abstract}
A parameterization of mesoscale eddy fluxes in the ocean should be consistent with the fact that the ocean interior is nearly adiabatic. Gent and McWilliams have described a framework in which this can be approximated in $z$-coordinate primitive equation models by incorporating the effects of eddies on the buoyancy field through an eddy-induced velocity. It is also natural to base a parameterization on the simple picture of the mixing of potential vorticity in the interior and the mixing of buoyancy at the surface. The authors discuss the various constraints imposed by these two requirements and attempt to clarify the appropriate boundary conditions on the eddy-induced velocities at the surface. Quasigeostrophic theory is used as a guide to the simplest way of satisfying these constraints.
\end{abstract}

\section{Introduction}

In ocean models used for climate studies requiring integrations over centuries to millennia, the incorporation of mesoscale eddy effects can only be achieved through parameterization, given the limitations of today's computers. In the present paper, we consider how parameterizations motivated by quasigeostrophic theory can be used to improve the treatment of eddy fluxes in primitive equation climate models. We work in the framework provided by Gent and McWilliams (1990), in which the effects of eddies on the buoyancy field in the nearly adiabatic ocean interior are accounted for through a three-dimensional "eddy-induced velocity." We discuss the appropriate boundary conditions for the eddy-induced velocity, the difficulty in diffusive theories of ensuring downgradient transport of potential vorticity and simultaneously conserving total buoyancy, and the associated dynamic constraints on the horizontal and vertical structure of the diffusivity.

Observations suggest that mesoscale variability is closely tied to the baroclinicity of the large-scale flow. Figure 1a shows the variance of sea level as obtained

Corresponding author address: Dr. Anne Marie Treguier, LPO, IFREMER, BP70, Plouzane 29280, France.

E-mail: treguier@ifremer.fr by TOPEX. Figure $1 \mathrm{~b}$ is a plot of a baroclinic time scale $T$, using the Levitus (1982) dataset. The growth rate of baroclinic waves in the classic Eady and Charney models scales as $f \mathrm{Ri}^{-1 / 2}$, where $f$ is the Coriolis parameter and

$$
\mathrm{Ri}=\frac{N^{2}}{\left(\partial_{z} U\right)^{2}+\left(\partial_{z} V\right)^{2}}
$$

is a Richardson number based on the large-scale mean flow $U, V$ and the mean stratification $N^{2}$. Held and Larichev (1996) argue that the vertical average of this timescale $T$ is representative of the timescale of the energy-containing eddies even when eddy amplitudes are much larger than mean shears:

$$
T^{-2}=f^{2}{\overline{\mathrm{Ri}^{-1}}}^{v} \text {. }
$$

We use this formula to generate Fig. 1b, with the vertical average $\overline{()}^{v}$ computed between $100 \mathrm{~m}$ and $2000 \mathrm{~m}$, using annual mean temperature and salinity fields, and computing the shears from geostrophy. The rough agreement between these two patterns encourages the idea that baroclinic production is a major source of eddy activity and that the relationship between baroclinicity and eddy statistics may be fairly local in the horizontal. Visbeck et al. (1997) recently used (1) in developing a parameterization and tested it successfully in a few idealized flow fields on an $f$ plane.

Quasigeostrophic (QG) theory provides a framework 

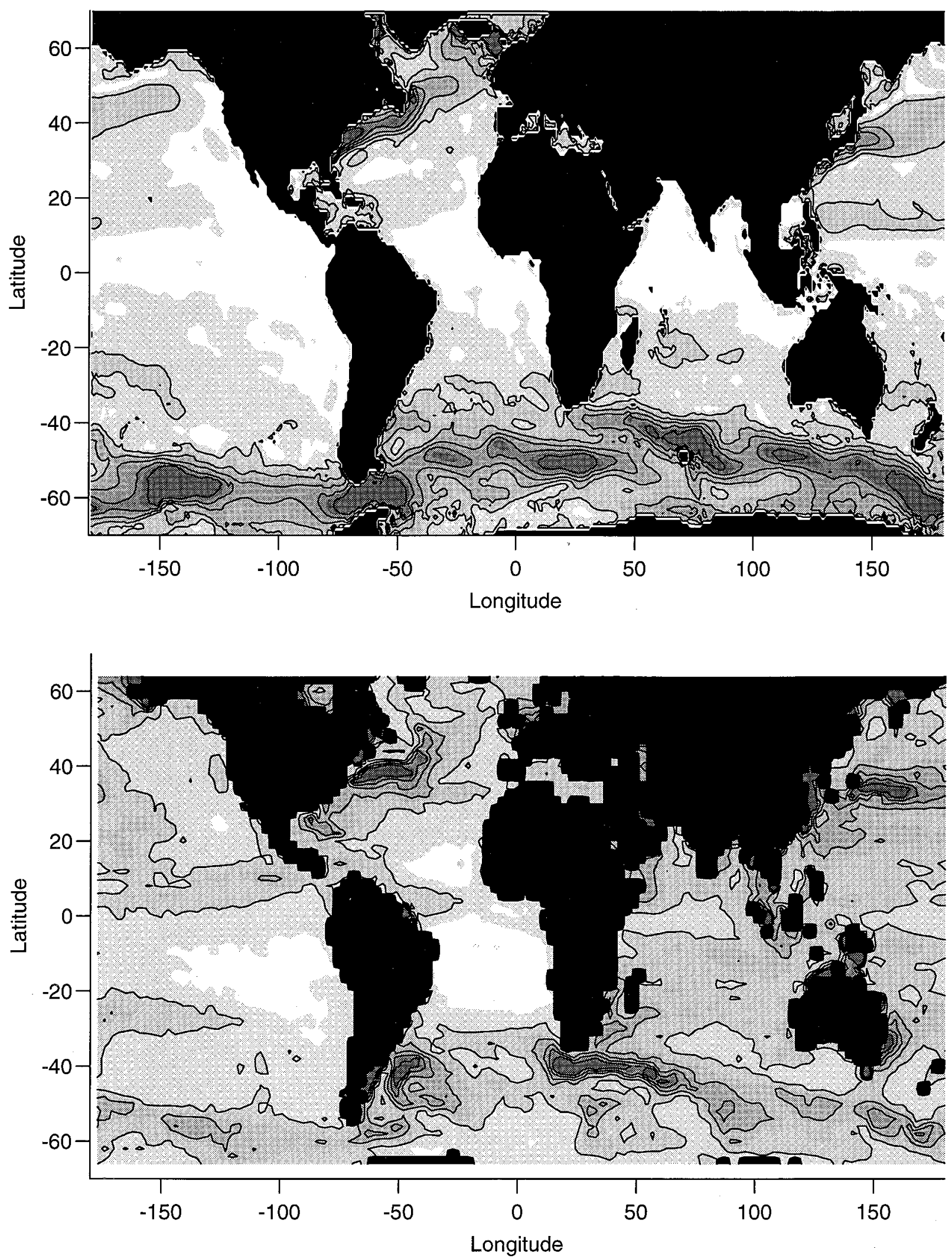

FIG. 1. (a: top) TOPEX rms sea-surface height variability averaged over 3 years (courtesy P. Y. Le Traon, Oceanography Group, C.L.S.). The grayscale marks intervals of $2.5 \mathrm{~cm}$ from 5 to $30 \mathrm{~cm}$. (b: bottom) Inverse timescale calculated according to (1) from the Levitus (1982) density field. The vertical integral is from 2000 to $100 \mathrm{~m}$. The grayscale marks intervals of $3 \times 10^{-7} \mathrm{~s}^{-1}$ from $3 \times 10^{-7} \mathrm{~s}^{-1}$ to $3 \times 10^{-6} \mathrm{~s}^{-1}$. 
for studying baroclinic eddy production. In the context of eddy flux parameterization, QG theory focuses attention on the eddy potential vorticity flux and on the horizontal fluxes of buoyancy at the ocean surface and bottom. Studies of eddy-mean flow interactions using QG models and concepts that are of particular relevance to the following discussion include Green (1970), Rhines (1977), Rhines and Holland (1979), Marshall (1981), Vallis (1988), and more recently Larichev and Held (1995), Pavan and Held (1996), and Visbeck et al. (1997).

We begin by discussing the transformed Eulerian mean (TEM) buoyancy equation and the eddy-induced velocity. The novel aspect of this discussion is the clarification of the boundary conditions at the ocean surface. We then motivate our focus on the buoyancy flux component of the potential vorticity flux at the expense of the relative vorticity flux. A brief review of factors that can create upgradient eddy fluxes is followed with a more detailed discussion of some difficulties in constructing a diffusive theory that 1) produces downgradient potential vorticity fluxes, 2) satisfies the kinematic constraint on the vertical integral of the potential vorticity flux, and 3) satisfies the requirement that these fluxes extract available potential energy from the basic flow. Some issues that arise when implementing parameterization schemes of the kind described herein are listed in the final section. We restrict the discussion to the buoyancy equation in a Boussinesq model. The treatment of tracer transport, including salinity and potential temperature, is discussed by Gent et al. (1995, hereafter GM95).

\section{Eddy-induced velocities}

We assume a separation between a mean flow $(\bar{\sigma})$ and eddies $\left(\sigma^{\prime}\right)$, where the mean may be considered as a time-mean and space average over a period and scale larger than the typical eddy scales, but smaller than climatic scales.

In isopycnal coordinates, the potential density equation and the continuity equation are combined into an equation for thickness, $\sigma=\partial z / \partial b$, where $b=-g \rho / \rho_{0}$ is the buoyancy and $z(x, y, b, t)$ is the height of a buoyancy surface. Following Rhines and Holland (1979), Andrews (1983), and Tung (1986), GM95 express the eddy flux of thickness averaged on an isopycnal surface as horizontal advection by the eddy-induced velocity $\mathbf{V} * *$ :

$$
\mathbf{V} * *=\frac{\overline{\mathbf{V}^{\prime} \sigma^{\prime}}}{\overline{\boldsymbol{\sigma}}^{b}},
$$

where $\bar{A}^{b}$ represents the average of $A$ following instantaneous isopycnals. The resulting mean thickness equation in an adiabatic region is simply

$$
\partial_{t} \overline{\boldsymbol{\sigma}}^{b}=-\boldsymbol{\nabla}_{b} \cdot\left[\left(\overline{\mathbf{V}}^{b}+\mathbf{V}^{* *}\right) \overline{\boldsymbol{\sigma}}^{b}\right],
$$

where $\nabla_{b}$ is the divergence taken on isopycnal surfaces.
An eddy flux closure in this framework consists of a theory for $\mathbf{V}^{* *}$. Any theory for this quantity will be consistent with the adiabatic character of the dynamics when used in (3). In particular, the mass between any two isopycnals will be conserved. One would like the $z$-coordinate model to share these features as closely as possible.

Transforming (3) back to height coordinates, one obtains

$$
\partial_{t} \bar{b}=-\boldsymbol{\nabla} \cdot\left[\left(\overline{\mathbf{V}}^{b}+\mathbf{V}^{* *}\right) \bar{b}\right]-\partial_{z}\left[\left(\bar{w}^{b}+w^{* *}\right) \bar{b}\right],
$$

where the vertical vorticities $\omega^{* *}$ and $\bar{\omega}^{b}$ are obtained from $\mathbf{V}^{* *}$ and $\overline{\mathbf{V}}^{b}$ using the continuity equation, and $\boldsymbol{\nabla}$ is the horizontal divergence. Note however that this equation is valid, not in $z$ coordinates but in what might be referred to as $\bar{z}$ coordinates, where $\bar{z}$ is obtained from the mean, rather than instantaneous buoyancy profile. By ignoring this difference, one can justify the use of advection by a three-dimensional velocity field as the parameterization of mesoscale eddies, with its horizontal component $\mathbf{V}^{* *}$ equal to the isopycnal thickness flux divided by the mean thickness (2).

This perspective leaves one with little guidance on how to treat the boundaries. As an alternative, one can start with the TEM buoyancy equation in $z$ coordinates. Following Andrews and McIntyre (1976) and Andrews et al. (1987), the conservation law for mean buoyancy in a Boussinesq model

$$
\partial_{t} \bar{b}+\overline{\mathbf{V}} \cdot \boldsymbol{\nabla} \bar{b}+\bar{\omega} \partial_{z} \bar{b}+\boldsymbol{\nabla} \cdot \overline{\mathbf{V}^{\prime} b^{\prime}}+\partial_{z} \overline{\omega^{\prime} b^{\prime}}=\bar{G}
$$

can be rearranged, without approximation, into its TEM form

$\partial_{t} \bar{b}+\left(\overline{\mathbf{V}}+\mathbf{V}^{*}\right) \cdot \boldsymbol{\nabla} \bar{b}+\left(\bar{w}+w^{*}\right) \partial_{z} \bar{b}=\bar{G}-\partial_{z} \Sigma$,

where

$$
\Sigma=\left[\frac{\overline{\mathbf{V}^{\prime} b^{\prime}} \cdot \nabla \bar{b}}{\partial_{z} \bar{b}}+\overline{w^{\prime} b^{\prime}}\right]
$$

and where the three-dimensional "eddy-induced velocity field," $\mathbf{V}^{*}$ and $w^{*}$, is defined as

$$
\omega^{*}=\boldsymbol{\nabla} \cdot \boldsymbol{\Psi}, \mathbf{V}^{*}=-\partial_{z} \Psi,
$$

where

$$
\boldsymbol{\Psi}=\frac{\overline{\mathbf{V}^{\prime} b^{\prime}}}{\partial_{z} \bar{b}} .
$$

All direct diabatic effects on the mean flow have been lumped into $G$. Here $\mathbf{V}$ and $\boldsymbol{\nabla}$ refer to the horizontal components of the velocity and gradient operator.

If the eddy fluxes are aligned along isopycnal surfaces,

$$
\overline{\mathbf{V}^{\prime} b^{\prime}} \cdot \boldsymbol{\nabla} \bar{b}=-\overline{w^{\prime} b^{\prime}} \partial_{z} \bar{b},
$$

then $\Sigma$ vanishes. If the interior of the ocean is adiabatic, $G=0$, it is tempting to argue that (10) holds so that

$\partial_{t} \bar{b}+\boldsymbol{\nabla} \cdot\left[\left(\overline{\mathbf{V}}+\mathbf{V}^{*}\right) \bar{b}\right]+\partial_{z}\left[\left(\bar{w}+w^{*}\right) \bar{b}\right]=0$, 
but this is not an exact result. More precisely, the budget for eddy buoyancy variance in the adiabatic interior is

$$
\begin{aligned}
\partial_{t}\left(\overline{\frac{b^{\prime 2}}{2}}\right)= & -\overline{\mathbf{V}^{\prime} b^{\prime}} \cdot \boldsymbol{\nabla} \bar{b}-\overline{w^{\prime} b^{\prime}} \partial_{z} \bar{b}-\overline{\mathbf{V}} \cdot \bar{\nabla} \frac{\overline{b^{\prime 2}}}{2} \\
& -\bar{w} \partial_{z} \frac{\overline{b^{\prime 2}}}{2}-\nabla \cdot \frac{\overline{\mathbf{V}^{\prime} b^{\prime 2}}}{2}-\partial_{z} \frac{\overline{w^{\prime} b^{\prime 2}}}{2} .
\end{aligned}
$$

The special case of this equation for a zonally uniform, small-amplitude wave train on a zonal flow is familiar from studies of linear instability:

$$
\partial_{t}\left(\overline{\frac{b^{\prime 2}}{2}}\right)=-\overline{v^{\prime} b^{\prime}} \partial_{y} \bar{b}-\overline{w^{\prime} b^{\prime}} \partial_{z} \bar{b} .
$$

If the wave is growing, (13) leads to the familiar result that the mixing slope for buoyancy is less steep than the mean isopycnals; when the wave is decaying, this slope is steeper than the isopycnals. For a statistically steady, adiabatic, linear wave field satisfying (13) the time-averaged eddy fluxes must lie along the mean isopycnals. However, in the oceans, and in the atmosphere for that matter, there is strong inhomogeneity of eddy statistics following the mean flow. The effect of the advection of variance by the mean flow in (12) in a statistically steady state is similar to that of transience in (13): if one can ignore the triple product representing advection of eddy variance by the eddies themselves, then, as one moves into a region of larger (smaller) variance following the mean flow, the mixing slope must be less steep (steeper) than the mean isopycnals. This issue is discussed in a recent paper by McDougall and McIntosh (1996).

At this point, one can try to fall back on a QG scaling argument. Quasigeostrophic theory cannot be applied to an entire ocean basin because of the limitations inherent in the standard theory with regard to the horizontal variations of the Coriolis parameter and the static stability (or isopycnal layer thickness). But one can hope that the local eddy dynamics can be approximated with QG theory so that it can provide useful insights into mesoscale flux parameterization. In QG theory, the buoyancy field $b$ is linearized about a reference profile with vertical gradient $N^{2}(z)$, which can be identified with the local value of $\partial_{z} \bar{b}$. In the QG limit the TEM buoyancy equation (6) reduces to

$$
\frac{\partial \bar{b}}{\partial t}+\overline{\mathbf{V}} \cdot \boldsymbol{\nabla} \bar{b}+\left(\bar{w}+w^{*}\right) N^{2}=\bar{G} .
$$

In this approximation the effect of horizontal eddy buoyancy fluxes is represented through the vertical advection of buoyancy by $w^{*}$. Horizontal advection by $\mathbf{V}^{*}$ is negligible in QG scaling, and $\Sigma$ is negligible as well, being of the order of the divergence of the vertical eddy buoyancy flux.

The isopycnal eddy thickness flux $\mathbf{V}^{* *}$ (2) is closely related to $\mathbf{V}^{*}$ in the QG limit as well. One has, first, the approximation

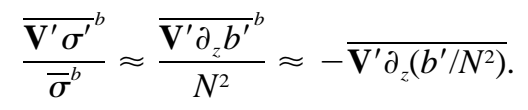

This relation follows from the general considerations in Charney and Stern (1962), who show that the dynamics of the QG (pseudo) potential vorticity on $z$ surfaces mirrors the dynamics of Ertel potential vorticity on isopycnals. [The lhs of (15) is related to the flux of Ertel potential vorticity on isopycnal surfaces, and the rhs to the flux of QG (pseudo) potential vorticity on $z$-surfaces.] In proving (15), one has to consider that an eddy defined on a $b$ surface is not identical to an eddy on a $z$ surface:

$$
A^{\prime}(x, y, z, t) \approx A^{\prime}\left(x, y, z_{0}, t\right)-\left.\frac{b^{\prime}}{\partial_{z} \bar{b}} \partial_{z} \bar{A}\left(z_{0}\right)\right|_{z=z_{0}},
$$

where $z_{0}$ is the height of an isopycnal in the mean state and $z$ is the actual height of that surface. In QG theory, this correction is needed for the stability perturbation, because it is assumed to be small compared to the mean stability, but not for the velocity itself. It follows from (15) and the thermal wind equation that

$$
\mathbf{V}^{* *} \approx \mathbf{V}^{*}+\frac{1}{f_{0}} \mathbf{k} \times \nabla \frac{\overline{b^{\prime 2}}}{N^{2}},
$$

where $f_{0}$ is the Coriolis frequency. The final term in (17) has zero horizontal divergence in QG theory, in which horizontal variations in $N^{2}$ are neglected. Therefore, $\boldsymbol{\nabla} \cdot \mathbf{V}^{* *} \approx \boldsymbol{\nabla} \cdot \mathbf{V}^{*}$, implying that $w^{* *} \approx w^{*}$. Since advection by $w^{*}$ is the dominant term in QG scaling, this is sufficient to show the sense in which (14) is the QG approximation to (4).

One might thereby be tempted to use advection by $w^{*}$ alone in the eddy flux closure, for consistency, but this would not provide a model that is adequately adiabatic in the interior. Following Gent and McWilliams (1990, hereafter GM90), we would like to retain the advection by $\mathbf{V}^{*}$, while continuing to assume $\Sigma=0$ as in (11), so as to achieve this goal.

McDougall and McIntosh (1996) provide a modification to the TEM transformation, somewhat analogous to a procedure used by Marshall and Shutts (1981) in another context (see section 6) that helps circumvent this difficulty. They show that by revising (9) to read

$$
\boldsymbol{\Psi}=\frac{\overline{\mathbf{V}^{\prime} b^{\prime}}}{\partial_{z} \bar{b}}-\frac{1}{\partial_{z} \bar{b}} \partial_{z}\left[\frac{\overline{\mathbf{V}} \overline{b^{\prime 2}}}{2 \partial_{z} \bar{b}}\right],
$$

the contribution to $\Sigma$ from the advection of variance by the mean flow in (12) is cancelled exactly. This creates a closer correspondence between the terms in the isopycnal (4) and TEM (11) balances, helping to justify the use of latter for eddy flux closure in $z$-coordinate models. In quasigeostrophic scaling, in which fractional changes in static stability are assumed to be small, this modification of $\boldsymbol{\Psi}$ would be neglected.

We will proceed under the assumption that the TEM buoyancy equation with $\Sigma=0$ in the ocean interior, 
and with $\boldsymbol{\Psi}$ approximated by (9) is adequate for mesoscale eddy flux closures. Given the present lack of theories for these eddy fluxes, it is difficult to imagine the distinction between (9) and (18) as being significant compared to the uncertainty in the closure itself.

It is worthwhile keeping in mind one curious point from the preceding discussion: the advection by $\mathbf{V}^{* *}$ in an isopycnal model is equivalent to three-dimensional advection by a nondivergent velocity field in a $z$-coordinate model; but vertical motion does not appear explicitly in the isopycnal formulation, while in the QG approximation (14), vertical advection $w^{*} \partial_{z} \bar{b}$ is dominant over horizontal advection, $\mathbf{V} * \boldsymbol{\nabla} \bar{b}$.

\section{Upper and lower boundary conditions}

The rigid-lid boundary condition for the mean vertical velocity is $\bar{w}=0$ at the surface $(z=0)$. However, $w^{*}$ is generally nonzero at the surface, as can be seen from its definition (8). The definition of $\omega^{*}$ is itself awkward near the surface due to the division by $\partial_{z} \bar{b}$, since $\partial_{z} \bar{b}$ will be close to zero in the mixed layer. We are therefore led to divide the domain into an interior region, within which we assume that the mesoscale eddies are essentially adiabatic, and surface layers, which contain the mixed layers in which turbulent mixing and air-sea heat exchange break this adiabatic constraint. The depths of these layers (at both the upper and lower boundaries) should be chosen somewhat larger than the depth of the mixed layers predicted by the large-scale model, due to the presence of the mesoscale eddy field, a point to which we return below. We distinguish in the following between the buoyancy fluxes due to mesoscale eddies and those resulting from the small-scale turbulence due to shear instabilities and convection. We assume that these physical processes act independently and that the small-scale processes are included in the forcing term $\bar{G}$ on the rhs of (5).

We must, first, require that $\overline{w^{\prime} b^{\prime}}$ vanish at the surface. Assuming that the mixing slope makes a smooth transition from the isopycnal slope in the adiabatic interior to its surface value, we write

$$
\overline{w^{\prime} b^{\prime}}=-\mu(z)\left(\frac{\overline{\mathbf{V}^{\prime} b^{\prime}}}{\partial_{z} \bar{b}} \cdot \nabla \bar{b}\right) .
$$

Here $\mu(z)$ is a function that passes from 1 to 0 as $z$ passes from the interior to the surface. Substituting this expression into (6), one finds

$$
\begin{aligned}
\partial_{t} \bar{b} & +\left(\overline{\mathbf{V}}+\mathbf{V}_{\mu}^{*}\right) \cdot \boldsymbol{\nabla} \bar{b}+\left(\bar{w}+w_{\mu}^{*}\right) \partial_{z} \bar{b} \\
& +(1-\mu) \boldsymbol{\nabla} \cdot \overline{\mathbf{V}^{\prime} b^{\prime}}=\bar{G},
\end{aligned}
$$

where $\left(\mathbf{V}_{\mu}^{*}, w_{\mu}^{*}\right)$ are obtained as in (8), but with

$$
\boldsymbol{\Psi}=\mu \frac{\overline{\mathbf{V}^{\prime} b^{\prime}}}{\partial_{z} \bar{b}} .
$$

Note that $w_{\mu}^{*}$ now vanishes at the surface. The formu- lation remains valid when $\mu$ (and the depth of the mixed layer) depend on $x, y$, and $t$.

We must still deal with the difficulty resulting from dividing by the stability in the surface layer. One alternative is to set $\mu$ to zero before reaching the mixed layer. But this has the awkward consequence of creating a strong horizontal eddy-induced mass flux immediately below the mixed layer. It is more physically plausible, and would cause fewer technical difficulties in models, to spread this horizontal mass transport through the entire surface layer. This can be achieved by setting

$$
\boldsymbol{\Psi}= \begin{cases}\frac{\overline{\mathbf{V}^{\prime} b^{\prime}}}{\partial_{z} \bar{b}}, & I_{-}<z<I_{+} \\ \left.\left(\frac{\overline{\mathbf{V}^{\prime} b^{\prime}}}{\partial_{z} \bar{b}}\right)\right|_{z=I_{+}} \mu(z), & I_{+}<z<0,\end{cases}
$$

where $I_{+}\left(I_{-}\right)$is the boundary between interior and the upper (lower) surface layer. An equation analogous to (23) would hold near the lower boundary as well. If there is a slope to the lower boundary, then $\mu$ should equal this slope at the boundary.

This treatment of the boundary condition has two important consequences. On the one hand, it introduces an eddy-induced horizontal mass flux in the upper and lower surface layers. In the upper surface layer, in particular, the magnitude of this mass flux is

$$
\mathbf{M}=\left.\left(\frac{\overline{\mathbf{V}^{\prime} b^{\prime}}}{\partial_{z} \bar{b}}\right)\right|_{z=I_{+}} \text {. }
$$

This mass flux appears as a surface $\delta$-function in quasigeostrophic theory (Bretherton 1966). By assuming that the mixing slope varies smoothly to its surface value, one spreads this mass flux over the surface layer. On the other hand, one also introduces horizontal eddy buoyancy fluxes into the surface layer through the term $(1-\mu) \boldsymbol{\nabla} \cdot \overline{\mathbf{V}^{\prime} b^{\prime}}$ in (20). The magnitude of the buoyancy tendency due to advection by this surface layer mass flux is $|\mathbf{M}||\boldsymbol{\nabla} b| / \delta$, where $\delta$ is the depth of the surface layer. The magnitude of the tendency due to the flux divergence in the surface layer is $\left|\overline{\mathbf{V}^{\prime} b^{\prime}}\right| / L$, where $L$ is the horizontal scale of the eddy buoyancy flux. Therefore, the ratio of the strengths of these two effects is

$$
\left.\frac{\text { advection }}{\text { mixing }} \approx \frac{L}{\delta} \frac{|\nabla b|}{\partial_{z} \bar{b}}\right|_{I} \approx \frac{L}{\delta} \alpha,
$$

where $\alpha$ is the slope of the isopycnals at the base of the surface layer. This ratio is dependent on the depth of this surface layer. If it is thin enough, eddy-induced advection dominates over mixing.

Various treatments of the boundary condition have been implemented in models that utilize the GM90 advective parameterization scheme. The approach described above can be compared with that used by Danabasoglu and McWilliams (1995). These authors use a diffusive parameterization for $\overline{V^{\prime} b^{\prime}}$, which does not van- 


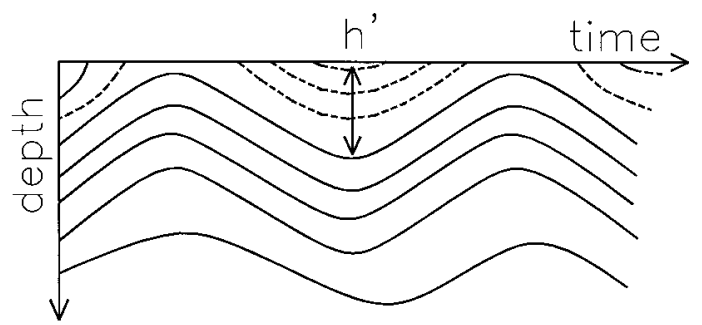

FIG. 2. Schematic evolution of the density field at a fixed point. Dashed isopycnals are not always present. The lowest densities are not even present in the time-mean state, but the mass flux $\overline{V^{\prime} h^{\prime}}$ associated with them does not vanish in general.

ish at the surface, but they effectively multiply this expression by the function $\mu(z)$. Although they do not discuss it in detail, the numerical scheme in Danabasoglu and McWilliams is equivalent to choosing $\delta$, the depth of the surface layer, equal to the depth of the top model level. This choice avoids the problem of dividing by $\left.\partial_{z} \bar{b}\right|_{\mathrm{m}}$ within the mixed layer, as long as the mixed layer is assumed to be contained within this surface layer, but this problem would emerge in a model in which the mixed layer is resolved. In addition, Danabasoglu and McWilliams do not include a parameterization of the divergence of the horizontal eddy buoyancy fluxes in the surface layer, implicitly assuming that the ratio in (25) is large.

Up to this point we have not assumed any particular form of closure; the fluxes need not be diffusive or even related to the local mean environment. However, in models in which, following GM90, temperature and salinity are advected by $\mathbf{V}^{*}$ and diffused along isopycnals, the diffusive effects on the buoyancy in the surface layer will be handled correctly by suppressing the isopycnal rotation of the mixing tensor near the surface.

The idea that horizontal buoyancy fluxes in the surface layer have these two distinct effects is unfamiliar, particularly with regard to the advection of buoyancy by a surface-layer eddy mass flux induced by the eddy buoyancy flux. It is useful to see how this mass flux arises in isopycnal models as well. Consider the evolution of the density field in an eddy-resolving isopycnal model at a fixed point (Fig. 2). New isopycnals (lighter fluid) can intermittently appear and disappear near the surface, for example, as a warm ring is advected past. They may not be present in the time-mean density field; however, their mass flux must be taken into account as part of an eddy closure scheme in a large-scale isopycnal model. In the atmosphere, this surface eddy flux is very important in midlatitudes, where most of the northward interior mass flux in warm potential temperature layers is returned as a southward surface flux in cold air outbreaks. The potential temperature of the air in these outbreaks is often lower than any potential temperature found in the mean state; nevertheless, these layers have to be taken into account in the global mass balance.

In an isopycnal model, one can proceed by distin- guishing between layers that rarely outcrop at the point in question and layers that appear only sporadically due to mesoscale variability. The mass flux $\mathbf{M}$ in the sum of the sporadic layers is related to the surface buoyancy flux in the limit of small displacements. Considering Fig. 2, the thickness variations $h^{\prime}$ of this layer can be approximated by $\pm b^{\prime} /\left(\partial_{z} \bar{b}\right)$ and

$$
\mathbf{M}_{ \pm}=\overline{\mathbf{V}^{\prime} h^{\prime}} \approx \pm \frac{\overline{\mathbf{V}^{\prime} b^{\prime}}}{\partial_{z} \bar{b}} .
$$

This is identical to the integrated surface layer flux in the $z$-coordinate model. If a mixed layer exists at the surface, then $\partial_{z} \bar{b}$ in this expression must be chosen as the stability beneath the mixed layer. This picture helps justify the choice (23) in a $z$-coordinate model. In an isopycnal model, an eddy closure would involve horizontal mixing of the buoyancy in this "surface layer" as well, in analogy with the $z$-coordinate model.

This isopycnal picture also suggests that the boundary between the interior and surface zones, which one is tempted to place at the base of the mixed layer in the $z$-coordinate model, should plausibly be lowered to the depth of the boundary between sporadic and uninterrupted isopycnal layers. Note that an increase in depth of the surface layer increases the importance of the surface eddy flux divergence as opposed to the eddy-induced advection in this layer.

\section{Quasigeostrophic eddy fluxes}

As is well known, the QG potential vorticity flux is the sum of two terms, a vorticity flux and a flux of "thickness":

$$
\overline{\mathbf{V}^{\prime} \zeta^{\prime}}+\overline{f \mathbf{V}^{\prime} \partial_{z}\left(\frac{b^{\prime}}{N^{2}}\right) .}
$$

The meridional component of the vorticity flux can be manipulated into the form

$$
\overline{v^{\prime} \zeta^{\prime}}=\partial_{x}\left(\overline{\frac{v^{\prime 2}-u^{\prime 2}}{2}}\right)-\partial_{y} \overline{u^{\prime} v^{\prime}}
$$

and similarly for the thickness flux

$$
f v^{\prime} \partial_{z}\left(\frac{b^{\prime}}{N^{2}}\right)=-f \partial_{x}\left(\overline{\frac{b^{\prime 2}}{2 n^{2}}}\right)+f \partial_{z}\left(\overline{\frac{v^{\prime} b^{\prime}}{N^{2}}}\right) .
$$

Analogous expressions exist for the zonal component of these fluxes. Equipartition between eddy kinetic and available potential energy implies

$$
u^{\prime} \approx v^{\prime} \approx b^{\prime} / N
$$

This scaling is typical of linear baroclinic instability, but also continues to hold in very strongly unstable flows (Larichev and Held 1995). If the eddy statistics only vary on horizontal scales much larger than $N h / f$, where $h$ is the vertical scale over which eddy statistics 
vary, it follows that the potential vorticity flux will be dominated by the thickness flux and that

$$
\overline{\mathbf{V}^{\prime} q^{\prime}} \approx f \partial_{z}\left(\overline{\frac{\mathbf{V}^{\prime} b^{\prime}}{N^{2}}}\right)
$$

Rhines and Holland (1979) offer a physical interpretation of this equation in terms of the form drag exerted by one isopycnal layer on neighboring layers.

If our large-scale model is not eddy-resolving, we can assume that its grid size is larger than $N h / f$, or, equivalently, at this level of approximation, larger than the deformation radius of the first baroclinic mode. Any eddy fluxes produced by a closure scheme imbedded in such a model can only vary on the larger resolved scales, so the use of (31) seems justified. However, this approximation can still break down if the eddies are more barotropic than implied by the equipartition assumption (30), as can occur if the source of the eddies is an essentially barotropic process. In particular, it is likely that the relative vorticity fluxes generated by barotropic mixing of potential vorticity $f / H$ near sharp gradients in the depth $H$ can play an important role in large-scale models, particularly near coasts (e.g., Holloway 1987). However, we suspect that it is useful at this time to separate this issue from that of the open-ocean eddy buoyancy fluxes. The following sections are written assuming that (31) is valid and, in references to "potential vorticity," the neglect of relative vorticity is always implied.

Given these approximations, a theory for the QG potential vorticity flux and surface buoyancy fluxes provides us with a theory for eddy-induced velocities. Comparing (31) and (8), we have

$$
\mathbf{V}^{*} \approx-f^{-1} \overline{\mathbf{V}^{\prime} q^{\prime}}
$$

in the interior and

$$
\mathbf{V}^{*} \approx-\left.f^{-1}\left(\frac{\overline{\mathbf{V}^{\prime} b^{\prime}}}{\partial_{z} \bar{b}}\right)\right|_{z=I_{ \pm}} \partial_{z} \mu(z)
$$

in the surface layers. One can then construct $w^{*}$ from the continuity equation. The simplest choice for $\mu$ of a linear function of $z$ results in a uniform distribution of $\mathbf{V}^{*}$ throughout the surface layers.

The approximation (31) leads to the following constraint on the QG potential vorticity flux in the adiabatic interior:

$$
\int_{I_{-}}^{I_{+}} \overline{\mathbf{V}^{\prime} q^{\prime}} d z=\left.\overline{f \frac{\mathbf{V}^{\prime} b^{\prime}}{N^{2}}}\right|_{I_{-}} ^{I_{+}} .
$$

In closures such as those discussed in section 5, in which potential vorticity is diffused in the interior and buoyancy is diffused at the surface, (34) imposes a strong constraint on the vertical structure of the diffusivity. Quasigeostrophic theory teaches us that density fluxes at the surfaces are a key part of the eddy-mean flow interaction and that they are intimately related to the interior potential vorticity fluxes. In contrast, if the closure is expressed in terms of the buoyancy fluxes themselves (for example, if one diffuses buoyancy in some way), then (34) is automatically satisfied.

We follow GM90 in transforming only the buoyancy equation, and not the momentum equations. In the meteorological literature (Andrews and McIntyre 1976) the momentum equations are generally transformed in an analogous way as for buoyancy, at least in the familiar application to zonally symmetric flows. This leads to an equation in which momentum is advected by, and the Coriolis force is computed with, the "total" velocity field, the mean flow plus the eddy-induced flow, $\left(\mathbf{V}_{T}\right.$, $w_{T}$ ), and in which the effects of eddies are accounted for through a "force" in the momentum equations. In the QG limit, the rotational part of this force is equal in strength, and in a direction perpendicular to, the potential vorticity flux itself. This suggests an alternative approach to the incorporation of eddy fluxes, in which the model flow variables are these "total" flows and one need only model the total potential vorticity flux by the eddies. The advantage of this approach is that the relative vorticity and buoyancy flux contributions to the potential vorticity flux can be treated in a unified way. However, we see no clear advantage to transforming the momentum equation unless relative vorticity fluxes are being modeled in some way and do not pursue this formulation here.

\section{Diffusive closures}

The intuition that eddy fluxes of a tracer should be directed down the mean tracer gradient is at least partly related to the presumption that there is a local balance between the generation and dissipation of eddy variance. In general, the QG eddy potential enstrophy balance reads as follows:

$$
\begin{aligned}
\partial_{t}\left(\overline{\left.\frac{q^{\prime 2}}{2}\right)=}\right. & -\overline{\mathbf{V}^{\prime} q^{\prime}} \cdot \nabla \bar{q}-\overline{\mathbf{V}} \cdot \nabla \frac{\overline{q^{\prime 2}}}{2} \\
& -\nabla \cdot \frac{\overline{\mathbf{V}^{\prime} q^{\prime 2}}}{2}-D
\end{aligned}
$$

where $D$, assumed to be positive, represents the dissipation on the small scales generated through an enstrophy cascade. This budget differs from the comparable variance budget for buoyancy in section 2 in two important ways: the inclusion of significant dissipation $D$ and the presence of two-dimensional rather than threedimensional advection. If one can ignore the advection of eddy variance by the mean flow and the self-advection of eddy variance described by the triple moment term, then, averaged over time, there must be a downgradient component to the potential vorticity flux at each location:

$$
\overline{\mathbf{V}^{\prime} q^{\prime}} \cdot \nabla \bar{q}=-D \text {. }
$$

If these advective terms are not negligible compared to 
the dissipation, one should not expect downgradient fluxes, nor should one expect a local relationship between fluxes and gradients.

In the special case of zonally symmetric mean flows with zonally symmetric eddy statistics, often considered as idealizations of the atmospheric general circulation, there is no advection of variance by the geostrophic flow, and one can confidently expect downgradient potential vorticity fluxes. In such flows, it appears that a local theory for the these fluxes can yield a very useful closure (see Pavan and Held 1996). But in the more general situation in which the variance has substantial variation along mean streamlines, one must expect upgradient fluxes in some regions.

This effect is especially important for potential vorticity because it is not a passive tracer. For example, Lau and Wallace (1979) point out the consequences of assuming that the eddies have a separable vertical structure (equivalent barotropic, in their terminology), $\mathbf{V}^{\prime}=$ $A(z) \mathbf{V}_{b}^{\prime}(x, y)$. The buoyancy and potential vorticity fluxes (continuing to ignore relative vorticity fluxes) can then be shown to circle regions of maximum streamfunction variance in an anticyclonic direction and to have no divergence. Under these circumstances, the fluxes have no special local relationship to the mean gradients. The divergence of the potential vorticity flux must be due to departures from this separable form. Since a single baroclinic mode is often found to dominate the eddy structure [e.g., during the MODE experiment, Richman et al. (1977)], one should expect a potential vorticity flux with a large rotational component in parts of the ocean.

Marshall and Shutts (1981) make a closely related point by assuming that the mean potential vorticity is approximately conserved following the mean flow so that there is a $\bar{q}-\bar{\psi}$ functional relationship, where $\bar{\psi}$ is the mean geostrophic streamfunction. They show that the eddy potential vorticity flux can then be divided into two parts, $\mathbf{F}_{1}+\mathbf{F}_{2}$, where the variance production due to $\mathbf{F}_{2}$ balances the advection of variance due to the mean flow. The consequence is that the production due to the remaining flux $\mathbf{F}_{1}$ balances the dissipation of variance $\mathrm{D}$ (as well as the triple moment, self-advection term), implying that $\mathbf{F}_{1}$ is more likely to be downgradient than the total flux $\mathbf{F}$. This is confirmed by a calculation of $\mathbf{F}_{1}$ and $\mathbf{F}_{2}$ in an eddy-resolving quasigeostrophic model by Marshall (1984). In addition, $\mathbf{F}_{2}$ can be defined as purely rotational, so removing this flux has no effect on eddy forcing of the mean buoyancy field.

The moral of these arguments is that one should not be discouraged by eddy-resolving models that show countergradient potential vorticity fluxes. The eddies may be predominantly equivalent barotropic, and there may exist a flux distribution that is everywhere downgradient that produces approximately the same forcing of the mean circulation. Buoyed by this argument, we feel that local theories in which the flux has a downgradient component at each location are an appropriate starting point for improving mesoscale eddy flux closures in ocean models. The comparison between the local baroclinicity of the oceans and eddy sea level variance in Fig. 1 provides further encouragement for a local theory based on the baroclinic production of eddies. In addition, any nonlocal closure should have a meaningful local closure at its core, valid in those cases when there is a local balance between eddy production and dissipation, and it is important to experiment with this local limit of the theory before adding the complexity of nonlocality.

It is natural to start with the simplest such local theory, in which the interior QG potential vorticity and the surface buoyancy fields are both diffused horizontally. In the interior

$$
\overline{\mathbf{V}^{\prime} q^{\prime}}=-\kappa(x, y, z) \nabla \bar{q},
$$

where

$$
\nabla \bar{q}=\beta \mathbf{j}+f \partial_{z}\left(\frac{\nabla \bar{b}}{\partial_{z} \bar{b}}\right),
$$

and where $\mathbf{j}$ is a unit vector in the $y$ direction. Consistent with our discussion of the scaling, this implies that

$$
\mathbf{V}^{*}=-\partial_{z}\left(\frac{\overline{\mathbf{V}^{\prime} b^{\prime}}}{\partial_{z} \bar{b}}\right)=\kappa\left[(\beta / f) \mathbf{j}+\partial_{z}\left(\frac{\boldsymbol{\nabla} \bar{b}}{\partial_{z} \bar{b}}\right)\right] .
$$

These expressions can be thought of as the $z$-coordinate QG version of the isopycnal mixing of Ertel potential vorticity $Q=f / \sigma$ :

$$
\overline{\mathbf{V}^{\prime} Q^{\prime}}{ }^{b}=-\kappa \nabla_{b} \bar{Q}^{b} .
$$

At the top and bottom boundary layers,

$$
\overline{\mathbf{V}^{\prime} b^{\prime}}=-\kappa_{b}(x, y) \nabla \bar{b},
$$

where the diffusivity for buoyancy, $\kappa_{b}$, need not be the same in the two surface layers.

The diffusivity has been assumed isotropic in the horizontal here, but this is arbitrary and presumably incorrect in detail even for those flows for which a local theory is adequate. In general, the diffusivity will have an asymmetric as well as a symmetric part, once again raising the issue of advective versus diffusive effects of mixing - and the symmetric, diffusive, part need not be isotropic. We tentatively suggest a departure from isotropic diffusion below.

This scheme differs from GM90, who set

$$
\mathbf{V}_{\mathrm{GM}}^{*}=\partial_{z}\left[\kappa \frac{\nabla \bar{b}}{\partial_{z} \bar{b}}\right] .
$$

First, the $\beta$ effect should be included in the environmental potential vorticity gradient. One would like an eddy flux closure to be able to create homogenized regions of potential vorticity, as in the numerical model of Holland and Rhines (1980) and as seen in observations (McDowell et al. 1982) rather than regions of uniform thickness. Admittedly, the spatial structure of $\kappa$ 
cannot be arbitrary in this formulation, because if one diffuses potential vorticity in a region with no baroclinicity, one will be creating isopycnal slopes where there were none originally, thereby creating rather than extracting available potential energy from the mean flow. A horizontally uniform $\kappa$ is unphysical in our context in which the eddy source is assumed to be baroclinic production. A related concern is that one must be wary of the singularity at the equator arising from the division by $f$. We consider these problems as providing physical constraints on the structure of $\kappa$, as described below, and not on the form of the equation.

Second, we have placed $\kappa$ outside, rather than inside, of the vertical derivative with the same motivation of staying as close as possible to potential vorticity mixing. The scheme (40) of GM90 has the feature that $\omega^{*}$ is local in $z$ :

$$
w_{\mathrm{GM}}^{*}=-\nabla \cdot\left[\kappa \frac{\nabla \bar{b}}{\partial_{z} \bar{b}}\right] .
$$

Using (39), we must obtain $\omega^{*}$ by a vertical integration. Since the baroclinic production of eddies involves the communication between different oceanic layers, there is no a priori reason to expect a closure that is local in $z$.

The difference between having $\kappa$ inside or outside the derivative is very clear in the QG limit, in which advection by $\omega^{*}$ dominates over that by $\mathbf{V}^{*}$ and the static stability can be assumed to be horizontally uniform. In the QG limit the GM90 closure reduces to horizontal diffusion of buoyancy in the interior:

$$
w^{*}{ }_{\mathrm{GM}} \partial_{z} \bar{b}=-\nabla \cdot(\kappa \nabla \bar{b}) .
$$

This relation should be kept in mind when comparing numerical experiments using the GM90 parameterization with experiments using horizontal mixing of density; the difference between two such experiments (which may be large on long time scales) is due only to the additional advection by $\mathbf{V}^{*}$ in GM and the influence of the boundary conditions. In an isopycnal model, this distinction is between an eddy flux based on the gradient of $z$ (the height of an isopycnal surface), rather than $\partial z / \partial \rho$ (the thickness).

These distinctions are also relevant when mixing coefficients are estimated from data or an eddy-resolving model. For example, in the meridional direction on an $f$ plane,

$$
\kappa(x, y, z) \approx-\left(\partial_{z} \frac{\overline{v^{\prime} b^{\prime}}}{\partial_{z} \bar{b}}\right) /\left(\partial_{y z} \frac{\bar{b}}{\partial_{z} \bar{b}}\right)
$$

is an estimate based on mixing of potential vorticity and compatible with (39), while

$$
\kappa_{\mathrm{GM}}(x, y, z) \approx-\overline{v^{\prime} b^{\prime}} / \partial_{y} \bar{b}
$$

is the coefficient based on GM90's assumptions. Ongoing experiments in a simple primitive equation jet model show that the diffusivity can have considerable structure in the vertical, so that the two estimates give very different results.

Finally, the diffusive closure as formulated here includes the horizontal diffusion of buoyancy in the top and bottom surface layers. The presence of downgradient horizontal buoyancy fluxes in the surface layer arises from the existence of air-sea fluxes, as discussed by Tandon and Garrett (1996), and also from the tendency of surface density to cascade to small scales (i.e., McWilliams and Gent 1994). If we ignore air-sea heat exchange for the moment, then within the QG approximation surface buoyancy satisfies the same equation as does potential vorticity near the surface. Therefore, surface buoyancy will cascade to small scales in the presence of mesoscale geostrophic turbulence, just as the potential vorticity is expected to do in the interior, with the variance eventually being removed at small scales. In this "nearly adiabatic" limit, one starts with the picture of surface buoyancy being diffused in the same way as near surface potential vorticity. The interior and boundary fluxes must be treated on an equal footing.

In the presence of damping due to air-sea heat exchange, this mixing of surface buoyancy will be modified; if the damping is strong enough, the eddy buoyancy perturbations will be reduced in magnitude and the mixing will also be reduced. Only in the unphysical limit of fixed surface buoyancy is there no horizontal eddy buoyancy flux at the surface.

Even with a diffusivity reduced from that in the interior, surface mixing remains of central importance since the mean gradient at the surface is strongly forced, so that there is always something to be diffused. In contrast, the interior potential vorticity gradient will be removed in regions of weak forcing, especially in unventilated layers, and the exact magnitude of the diffusivity will be of relatively little importance to the buoyancy field (Rhines and Young 1982). Especially with respect to the effects of eddies on the poleward heat transport, we expect that the surface diffusion may be a very important part of such a closure scheme (Drijfhout 1994).

\section{Constraints on the diffusivity}

Two important constraints must be satisfied by the diffusivities, both of which exist because potential vorticity is not a passive tracer. First, the resulting fluxes should be kinematically consistent, satisfying (34) in the vertical column at each horizontal point. From the perspective of the induced eddy velocity, this condition is needed to ensure that $w^{*}$ in the interior can match the flux divergence in both the upper and lower surface layers so that total buoyancy is conserved. Stated differently, one must be able to consistently generate an eddy buoyancy flux at each point by integrating the potential vorticity flux in the vertical.

Second, the fluxes should extract available potential energy from the mean flow, also at each point since we 
are attempting to mimic the consequences of local baroclinic eddy production. The expression of this constraint is $P>0$, where

$$
P=-\int_{I_{-}}^{I_{+}} \frac{\overline{\mathbf{V}^{\prime} b^{\prime}} \cdot \boldsymbol{\nabla} \bar{b}}{\partial_{z} \bar{b}} d z .
$$

We integrate only over the interior adiabatic layer for convenience, but the surface layers are presumed to be thin anyhow. Using the thermal wind equation, $\nabla \bar{b}=$ $-f \partial_{z} \overline{\mathbf{W}}$, where $\overline{\mathbf{W}}=\mathbf{k} \times \overline{\mathbf{V}}$, and integrating by parts,

$$
P=\int_{I_{-}}^{I_{+}} \overline{\mathbf{V}^{\prime} q^{\prime}} \cdot \overline{\mathbf{W}} d z-\left.f\left(\overline{\frac{\mathbf{V}^{\prime} b^{\prime}}{\partial_{z} \bar{b}}} \overline{\mathbf{W}}\right)\right|_{I_{-}} ^{I_{+}} .
$$

If (34) is satisfied, then adding a constant to the flow field leaves (46) unmodified, in which case one can set the barotropic component of the flow equal to zero in this expression.

We have no theory for the structure of the diffusivity, but we can tentatively propose some more or less arbitrary ways in which these constraints can be satisfied. We start by focusing on the requirement that the energy production be positive. We choose a separable form for the diffusivity

$$
\kappa(x, y, z)=\kappa_{h}(x, y) \gamma(z),
$$

where the maximum value of $\gamma$ is unity, so that $\kappa_{h}$ is the maximum diffusivity in the water column. This form allows us to discuss the questions of horizontal and vertical structure of the diffusivity separately. We assume for simplicity that surface buoyancy is diffused with the same diffusivity as potential vorticity near the surface. The rate of baroclinic energy production (46) is then $P=\kappa_{h} / T_{\text {eff }}^{2}$, where

$$
T_{\text {eff }}^{-2}=-\int_{I_{-}}^{I_{+}} \gamma(z) \boldsymbol{\nabla} q \cdot \overline{\mathbf{W}} d z+\left.f \gamma \frac{\boldsymbol{\nabla} b \cdot \overline{\mathbf{W}}}{\partial_{z} \bar{b}}\right|_{I_{-}} ^{I_{+}} .
$$

If the diffusivity is independent of $z(\gamma=1)$ and if $\beta$ is negligible so that

$$
\nabla q=f \partial_{z}\left(\frac{\nabla \bar{b}}{\partial_{z} \bar{b}}\right),
$$

then $T_{\text {eff }}=T$ defined by (1), as can be seen by integrating by parts again, so that

$$
T_{\mathrm{eff}}^{-2}=\int_{I_{-}}^{I_{+}} \frac{\boldsymbol{\nabla} \bar{b} \cdot \nabla \bar{b}}{\partial_{z} \bar{b}} d z(\gamma=1) .
$$

Following Larichev and Held (1995), we balance this production with the rate of the barotropic inverse energy cascade, $V^{3} k_{0}$, where $V$ is the rms barotropic velocity and $k_{0}$ the wavenumber of the energy-containing scale. Balancing production and this cascade rate and assuming $\kappa_{h} \approx V k_{0}^{-1}$ leads to the estimate, $T_{\text {eff }} \approx\left(k_{0} V\right)^{-1}$ and

$$
\kappa_{h} \approx \frac{1}{k_{0}^{2} T_{\text {eff }}} \text {. }
$$

This construction provides an expression for $\kappa_{h}$ consis- tent with the scaling arguments in Larichev and Held (1995) for the very strongly unstable limit in which the diffusivity is independent of depth. Held and Larichev (1996) also argue that, if the inverse cascade is halted by the $\beta$ effect, then $k_{0} \approx \beta T$, but the relevance of this scaling for the oceans is unclear and we leave the choice of $k_{0}$ open. In the case that $k_{0}$ is chosen to be independent of the vertical shear, this scheme is equivalent to that proposed by Green (1970) and implemented recently by Visbeck et al. (1997).

These arguments lead to the following proposal: given the vertical structure of the diffusivity $\gamma(z)$, compute $T_{\text {eff }}^{-1}$ from (48). If the result is negative production, then one can set $T_{\text {eff }}^{-1}=0$. Given, in addition, an expression for $k_{0}$, compute $\kappa_{h}$ from (51). This procedure has the property of ensuring that the diffusivity vanishes smoothly as the implied energy production decreases to zero.

The kinematic consistency condition (34) presents a constraint of the vertical structure of the diffusivity that is more difficult to satisfy, especially if $\beta$ is not ignored in the potential vorticity gradient. In terms of the diffusion coefficients, this constraint is

$$
\int_{I_{-}}^{I_{+}} \gamma(z) \nabla \bar{q} d z=\left.\left(\gamma \frac{\nabla \bar{b}}{\partial_{z} \bar{b}}\right)\right|_{I_{-}} ^{I_{+}} .
$$

We have again made the simplifying assumption that the diffusivity for buoyancy at the boundaries is equal to the diffusivity for potential vorticity near the boundaries.

This is a local constraint for the water column at each horizontal location. This constraint is local because of our neglect of the contribution of relative vorticity to the potential vorticity flux. If the relative vorticity is not neglected, one is only left with a global constraint on the three-dimensional integral of the potential vorticity flux. But this is an illusory freedom; if (52) is not satisfied locally, one will be generating large relative vorticity fluxes that are inconsistent with the scaling (30), so these fluxes will be unphysically large. At this level of approximation, we feel that it is safer and more consistent to neglect the relative vorticity fluxes and try to satisfy this local constraint.

One simple expedient is to choose $\gamma=1$, a diffusivity independent of depth. This will automatically satisfy (52), but only if one ignores the contribution of $\beta$ to the potential vorticity gradient (49) and diffuses surface buoyancy and the interior potential vorticity with the same strength. Countergradient potential vorticity fluxes might be created by this scheme due to the neglect of $\beta$, but only in weakly unstable regions. In the strongly unstable regions in which the diffusivities are the largest, $\beta$ may play a small role. In addition, as discussed by Larichev and Held (1995), diffusivities should become more and more uniform with depth as the flow becomes more unstable. Therefore, a diffusivity that is uniform in the vertical may be a useful simplification, 
despite the distortion of the eddy fluxes in weakly unstable regions that would result. This simplification has been adopted with success by Visbeck et al. (1997).

Moving beyond a diffusivity that is uniform in the vertical is surprisingly difficult. It may be useful in this context to see how a linear unstable mode satisfies (52) while producing downgradient potential vorticity fluxes, an approach used by Green (1970). In principle, one could take the vertical structure of the flow at each point and formulate the pure QG baroclinic instability problem, ignoring horizontal shears. The flux in an unstable mode must have a downgradient potential vorticity flux at each $z$ and will automatically satisfy (52), so one can compute a consistent $\gamma(z)$. If the flow is stable, the diffusivity can be set to zero. Even if one trusted the vertical structure from the most unstable mode, say, this algorithm is unlikely to be practical as it would presumably be too time consuming to solve an eigenvalue problem whenever the diffusivities were needed. The problem would then be to replace the explicit calculation with an approximate theory that continues to satisfy these constraints. We do not have such a theory.

A more satisfying approach, but far more impractical yet, would be to integrate a homogeneous turbulence model of the sort treated by Larichev and Held (1995), for each point (!) in the large-scale model, by prescribing the appropriate environmental current structure as a function of depth. The potential vorticity fluxes produced by this model will have a downgradient component [the model is homogeneous by construction, so there is no advection of variance in (35)] and would provide a consistent local eddy closure. (One could even argue that this is the only appropriate local closure "theory.") But the problem of parameterization is that of developing approximations for the fluxes produced by this homogeneous model.

We can try to proceed more heuristically. Consider first the case of a plane-parallel (zonal) mean flow, for which we are only concerned with the meridional component of (52). We can then use the expedient of formulating a plausible set of vertical structures $\gamma(z, \mu)$ dependent on a parameter $\mu$, choosing $\mu$ so that the meridional component of (52) is satisfied. This procedure has the useful property that, if $\partial_{y} \bar{q}$ is of one sign throughout the column and if the buoyancy gradients are of the signs required by the Charney-Stern-Pedlosky criterion for baroclinic stability (buoyancy decreasing poleward at the upper boundary and increasing poleward at the lower boundary), then we will not be able to find a value of $\mu$ that will satisfy this constraint (with positive $\gamma$ for all $z$ ) and would set the diffusivity to zero. A related approach has been used by White and Green (1984) in an atmospheric context.

In the appendix, we try to motivate a particular choice for this set of vertical structures:

$$
\gamma \propto\left(1+\mu \bar{u}_{r}\right)^{2}
$$

where $\bar{u}_{r}$ is the baroclinic component (vertical mean removed) of the zonal flow. This expression has the intuitive property of generating a surface-intensified diffusivity for the case of a surface-intensified baroclinic jet. It seems to work well in predicting the vertical structure of the potential vorticity flux in some multilayer homogeneous turbulence simulations (Larichev), but we do not expect it to work well for all possible flows. For weakly unstable flow, in particular, mixing will be concentrated near critical lines, where the dominant phase speed of the eddies equals the mean current, and one would be better off using a linear theory to estimate this phase speed. The choice (53) is meant to apply to those regions where the shears are strong enough to generate eddies with a substantial barotropic component.

If the mean potential vorticity gradients change direction with height, then the kinematic constraint (52) is a vector equation that is more difficult to satisfy. In general, it does not appear possible to satisfy this constraint while restricting oneself to isotropic diffusion. One can see this by examining the simplest horizontally homogeneous two-layer model of baroclinic instability, for which the potential vorticity flux vectors in the two layers must be equal and opposite, but the potential vorticity gradients in the two layers need not be parallel if the gradient of the interface height is not directed north-south. In this kind of flow, unstable baroclinic modes will produce potential vorticity fluxes that have a downgradient component, but they cannot be oriented exactly downgradient. This example suggests the option of assuming that the the eddies are "polarized" so that all eddy flux vectors in a particular water column at different heights are parallel or antiparallel. The problem then reduces to the plane-parallel case described above. But one is also left with the difficulty of choosing this distinguished direction at each horizontal location. Perhaps the structure of the unstable baroclinic modes can be used as guidance in this regard. In addition, the expression (48) for $T_{\text {eff }}$ would have to be modified appropriately if the diffusivity is not isotropic.

\section{Implementation}

Several questions arise in the implementation of this diffusive potential vorticity mixing in existing primitive equation ocean models. We list several of these.

1) One must define the boundaries between the adiabatic interior and the surface layers, in which the buoyancy fluxes are not directed along the mean buoyancy surfaces. In order to avoid dividing by a zero static stability in the definition of the potential vorticity gradient, these surface layers should, at the minimum, contain the mixed layers generated by the model's parameterization of convective or winddriven mixing. While it can be argued that the presence of mesoscale eddies will effectively deepen these layers, this is difficult to quantify, and it may be adequate to place this boundary at the first vertical 
velocity grid point interior to the mixed region. This boundary would then move in time.

2) For the horizontal structure of the diffusivity, we suggest using the separable form (47) with $\kappa_{h}$ given by (51), with the timescale $T_{\text {eff }}$ defined by (48). The choice of mixing length $k_{0}^{-1}$ has been left open, however. This choice can have profound effects of the horizontal structure of the diffusivity. A crucial issue is whether this scale is determined by 1) some Rossby deformation radius (as in the theory of Stone 1972), by 2) horizontal inhomogeneities of the mean flow such as the "width" of a strongly baroclinic zone (as in the theory of Green 1970 and as implemented by Visbeck et al. 1997), or by 3) the process that stops the inverse barotropic energy cascade, such as the topographic or planetary beta-effect, as in Held and Larichev (1996). The homogeneous simulations of Larichev and Held (1995) and Held and Larichev suggest an appropriate choice is the smaller of 2) and 3). However, we note that a choice of a mixing length larger than the grid size is (at least intuitively) inconsistent with the assumption of a local relationship between fluxes and gradients.

3) The choice of vertical structure is even more problematic. The simplest alternative is to assume that the diffusivity is independent of depth, while ignoring the contribution of beta to the potential vorticity gradients. Indeed, we have no explicit proposal for an alternative that is workable in the general case of nonparallel potential vorticity gradients, that would be encountered within a large-scale model. It is not easy to simultaneously satisfy the two constraints of a potential vorticity flux that is everywhere downgradient and an eddy-induced velocity that conserves buoyancy. In fact, in the general case in which currents change direction with depth, it does not appear natural to try to satisfy these constraints with isotropic diffusion. We have suggested one alternative - the assumption of "polarized eddies" that produce a flux in the same direction at all levels, but we cannot advocate this strongly without further study.

4) One must choose diffusivities for buoyancy in the two surface layers, as well as for the interior potential vorticity. The simplest assumption is that these surface diffusivities are equal to the values used for potential vorticity at the internal boundaries of these layers. One expects the surface diffusivities to be smaller, due to the damping arising from air-sea energy exchange, but estimating this reduction introduces additional complexities. In the special case in which one assumes a diffusivity that is uniform in the vertical, one is constrained to diffuse surface buoyancy with this same coefficient.

\section{Concluding remarks}

The advective parameterization of mesoscale eddies in climate models has been considered from a quasi- geostrophic point of view. We hope that our presentation complements those of Gent et al. (1995) and Tandon and Garrett (1996), who have recently addressed many of the same issues. We have attempted to clarify the boundary condition for the eddy-induced velocity at the ocean surface and have stressed that the horizontal flux of surface buoyancy has two distinct effects in this context: the creation of eddy-induced mass fluxes in the surface and bottom boundary layers, and a horizontal mixing of surface buoyancy. We have discussed the differences between the diffusive eddy flux closures based on downgradient transport of potential vorticity/ surface buoyancy, as motivated by quasigeostrophic dynamics, and diffusion of interior buoyancy. We have also focused on the constraints imposed on the diffusivity if one desires these fluxes to be physically realizable by some (quasigeostrophic) field of eddies and if one requires the eddy-induced velocities to satisfy natural boundary conditions at the upper and lower boundaries and conserve total buoyancy.

Data clearly show the inhomogeneity of the oceanic eddy activity. Non-eddy-resolving climate models have evolved to the point where this inhomogeneity has to be taken into account, moving beyond globally uniform diffusion coefficients. Horizontally variable and flowdependent diffusion coefficients appear to be a reasonable next step, even if not fully satisfactory. Taking this step should lead to significant improvements in climate models in the near future, as the idealized models of Visbeck et al. (1997) suggest.

Different issues arise concerning the horizontal and vertical structures of a variable diffusivity. With regard to the horizontal structure, if one assumes that the relevant eddies are generated by local baroclinic production, it seems appropriate to define a mixing coefficient depending on some depth-averaged baroclinicity of the large-scale flow, following the parameterization proposed by Held and Larichev (1996), for example. We have described how this approach naturally ensures that diffusion coefficients will vanish in regions in which energy cannot be extracted from the mean available potential energy reservoir. This approach should be contrasted with Smagorinsky-type mixing schemes, in which the strength of the diffusion is a function of the horizontal shears or deformation, rather than vertical shears of the mean flow, and the source of eddy energy is implicitly the mean kinetic, rather than available potential, energy.

The major source of uncertainty in the construction of such models concerns the vertical structure of the diffusivity for potential vorticity. The difficulty is that a closure theory for the eddy-induced circulation (8) must be consistent with the fact that $\mathbf{V}^{*}$ is itself the vertical derivative of $\overline{\mathbf{V}^{\prime} b^{\prime}} / \partial_{z} \bar{b}$. If one uses a closure theory for $\overline{\mathbf{V}^{\prime} b^{\prime}}$ itself, there is no difficulty in conserving buoyancy, but one can then generate countergradient potential vorticity fluxes. If one diffuses potential vorticity instead, but with an arbitrary vertical structure, 
one will be assured of downgradient transport, but one will not conserve buoyancy unless the diffusivities are appropriately constrained. In the general case, this apparently requires a horizontally anisotropic isopycnal diffusivity.

A fundamental issue related to the validity of closure schemes of the sort considered here is that the available potential energy extracted from the mean flow is presumed to be converted to eddy (kinetic plus potential) energy and then dissipated, rather than returned to the kinetic energy of the larger scale flow, as might be anticipated in a weakly dissipative system. Schemes with conservative energetics can be constructed by moving away from simple diffusion, as in the "anticipated potential vorticity mixing" approach of Sadourny and Basdevant (1985). Alternatively, one can imagine closure schemes that contain appropriately designed stochastic forcing on the smallest resolved scales, so as to generate kinetic energy on these scales equal in magnitude to the loss of available potential energy.

We have not touched upon questions of "cross-isopycnal" mixing. Even if the cross-isopycnal mixing is not directly due to mesoscale eddies, the smaller scales that are responsible could still be organized by the mesoscale eddy field, so that the cross-isopycnal mixing coefficient could depend on one's parameterization of eddy activity (Tandon and Garrett 1996). In any case, the first step is to develop plausible theories for eddy scales and energy levels on the mesoscale.

Given these uncertainties, confidence in any closure scheme will have to grow piecemeal through experimentation with realistic global models and comparison with eddy-resolving models of various levels of complexity.

Acknowledgments. This paper was written while A. M. Treguier enjoyed an extended visit at GFDL, with support from C.N.R.S. and the A.O.S. program of Princeton University. The views expressed herein are those of the authors and do not necessarily reflect the views of NOAA or any of its subagencies.

\section{APPENDIX}

\section{Vertical Structure of the Diffusivity}

For a very strongly unstable flow that produces a substantial inverse energy cascade, the eddies produced should be nearly barotropic and the resulting eddy diffusivity should be approximately independent of $z$. To motivate a choice of a vertical structure for the diffusivity in less strongly unstable situations, we perturb away from this nearly barotropic limit. Assuming that the barotropic component is still dominant, the baroclinic component of the potential vorticity generated by the barotropic eddy flow will then be of the order of

$$
q_{\tau}^{\prime} \approx-(\partial \bar{q} / \partial y)_{\tau} k_{0}^{-1},
$$

where the mixing length $k_{0}^{-1}$ is independent of height.
The subscript $\tau$ refers to the deviation from a vertical mean. With similar expression for the buoyancy at the upper and lower boundaries we find that

$$
\psi_{\tau}^{\prime} \approx \bar{u}_{\tau}(z) k_{0}^{-1}
$$

and

$$
v_{\tau}^{\prime} \approx \bar{u}_{\tau}(z)
$$

For related arguments see Larichev and Held (1995).

We now assume that $\kappa$ has the vertical structure of the total eddy velocity variance $\overline{v^{\prime 2}}$, which, in turn, is written as the coherent sum of the constant barotropic velocity $V$ and the baroclinic velocity as estimated in (A3):

$$
\begin{gathered}
\overline{v^{\prime 2}} \approx V^{2}\left(1+\mu \frac{\bar{u}_{r}}{V}\right)^{2} \\
\kappa \propto\left(1+\mu \overline{u_{r}}\right)^{2} .
\end{gathered}
$$

Here $\mu$, or $\mu^{*}$, is a constant, independent of $z$ introduced to take into account the correlations between the barotropic and baroclinic components of the flow and to provide the freedom to satisfy (52). If the flow satisfies the Charney-Stern criterion for stability, one will not be able to satisfy the constraint (52) whatever the form chosen for the diffusivity, as long as it is positive definite.

\section{REFERENCES}

Andrews, D. G., 1983: A finite-amplitude Eliassen-Palm theorem in isentropic coordinates. J. Atmos. Sci., 40, 1877-1883.

_, and M. E. McIntyre, 1976: Planetary waves in horizontal and vertical shear: The generalized Eliassen-Palm relation and the mean zonal acceleration. J. Atmos. Sci., 33, 2031-2048.

_ - J. R. Holton, and C. B. Leovy, 1987: Middle Atmosphere Dynamics. Academic Press, 489 pp.

Bretherton, F. P., 1966: Critical layer instability in baroclinic flows. Quart. J. Roy. Meteor. Soc., 92, 325-334.

Charney, J. G., and M. E. Stern, 1962: On the stability of internal baroclinic jets in a rotating atmosphere. J. Atmos. Sci., 19, 159172 .

Danabasoglu, G., and J. C. McWilliams, 1995: Sensitivity of the global ocean circulation to parameterizations of mesoscale tracer transports. J. Climate, 8, 2967-2987.

Drifjhout, S. S., 1994: Sensitivity of eddy-induced heat transport to diabatic forcing. J. Geophys. Res., 99, 18 481- 18499.

Gent, P. R., and J. C. McWilliams, 1990: Isopycnal mixing in ocean circulation models. J. Phys. Oceanogr., 20, 150-155.

, J. Willebrand, T. J. McDougall, and J. C. McWilliams, 1995: Parameterizing eddy-induced tracer transports in ocean circulation models. J. Phys. Oceanogr., 25, 463-474.

Green, J. S. A., 1970: Transfer properties of large scale eddies and the general circulation of the atmosphere. Quart. J. Roy. Meteor. Soc., 96, 157-185.

Held, I. M., and V. D. Larichev, 1996: A scaling theory for horizontally homogeneous, baroclinically unstable flow on a $\beta$-plane. J. Atmos. Sci., 53, 946-952.

Holland, W., and P. B. Rhines, 1980: An example of eddy-induced ocean circulation. J. Phys. Oceanogr., 10, 1010-1031.

Holloway, G., 1987: Systematic forcing of large scale physical flows by eddy-topography interaction. J. Fluid Mech., 184, 463-476.

Larichev, V. D., and I. M. Held, 1995: Eddy amplitudes and fluxes 
in a homogeneous model of fully developed baroclinic instability. J. Phys. Oceanogr., 25, 2285-2297.

Lau, N. C., and J. M. Wallace, 1979: On the distribution of horizontal transports by transient eddies in the Northern Hemisphere wintertime circulation. J. Atmos. Sci., 36, 1844-1861.

Levitus, S., 1982: Climatological Atlas of the World Ocean. NOAA Prof. Paper No. 13, U.S. Govt. Printing Office, 174 pp.

Marshall, J. C., 1981: On the parameterization of geostrophic eddies in the ocean. J. Phys. Oceanogr., 11, 257-271.

$\longrightarrow$, 1984: Eddy-mean-flow interaction in a barotropic ocean model. Quart. J. Roy. Meteor. Soc., 110, 573-590.

—, and G. J. Schutts, 1981: A note on rotational and divergent fluxes. J. Phys. Oceanogr., 11, 1677-1680.

McDougall, T. J., and P. C. McIntosh, 1996: The temporal-residualmean velocity. Part I: Derivation and the scalar conservation equations. J. Phys. Oceanogr., 26, 2653-2665.

McDowell, S., P. B. Rhines, and T. Keffer, 1982: North Atlantic potential vorticity and its relation to the general circulation. $J$. Phys. Oceanogr., 12, 1417-1436.

McWilliams, J. C., and P. Gent, 1994: The wind-driven ocean circulation with an isopycnal-thickness mixing parameterization. $J$. Phys. Oceanogr., 24, 46-65.

Pavan, V., and I. M. Held, 1996: The diffusive approximation for eddy fluxes in baroclinically unstable jets. J. Atmos. Sci., 53, $1262-1272$.

Plumb, R. A., and J. D. Mahlman, 1987: The zonally averaged transport characteristics of the GFDL general circulation/transport model. J. Atmos. Sci., 44, 298-327.
Rhines, P. B., 1977: The dynamics of unsteady currents. The Sea, Vol. 6, Marine Modelling, E. D. Goldberg, I. N. McCane, J. J. O'Brien, and J. H. Steele, Eds., Wiley-Interscience, 189-318.

- , and W. R. Holland, 1979: A theoretical discussion of eddydriven mean flows. Dyn. Atmos. Oceans, 3, 289-325.

—, and W. R. Young, 1982: Homogenization of potential vorticity in planetary gyres. J. Fluid Mech., 122, 347-367.

Richman, J. G., C. Wunsch, and N. Hogg, 1977: Space and time scales of mesoscale motion in the sea. Rev. Geophys. Space Phys., 15, 385-420.

Sadourny, R., and C. Basdevant, 1985: Parameterization of subgridscale barotropic and baroclinic eddies in quasi-geostrophic models: Anticipated vorticity method. J. Atmos. Sci., 42, 1353-1363.

Stone P., 1972: A simplified radiative-dynamical model for the static stability of rotating atmospheres. J. Atmos. Sci., 29, 405-418.

Tandon, A., and C. Garrett, 1996: On a recent parameterization of mesoscale eddies. J. Phys. Oceanogr., 26, 406-411.

Tung, K. K., 1986: Nongeostrophic theory of zonally averaged circulation. Part I: Formulation. J. Atmos. Sci., 43, 2600-2618.

Vallis, G. K., 1988: Numerical studies of eddy transport in eddyresolving and parameterized models. Quart. J. Roy. Meteor. Soc., 114, 183-204.

Visbeck, M., J. Marshall, T. Haines, and M. Spall, 1997: On the specification of eddy transfer coefficients in coarse-resolution ocean circulation models. J. Phys. Oceanogr., 27, 381-402.

White, A. A., and J. S. A. Green, 1984: Transfer coefficient eddy flux parameterization in a simple model of the zonal average atmospheric circulation. Quart. J. Roy. Meteor. Soc., 110, 10351052 . 